\title{
Stressregulation durch Gesundheitsstärkung
}

Sport und Bewegung beeinflussen den Stressprozess auch von seinem Ende her, also von den gesundheitlichen Konsequenzen einer mehr oder weniger gelungenen Stressverarbeitung (Pfad 4 in Abb. 2.1). Angenommen wird hier, dass Menschen, die durch ihre regelmäßige körperliche Aktivität über eine gute Gesundheit verfügen, längere Zeit unter hohem Stress stehen können, ohne einen physischen oder psychischen Einbruch zu erleiden. De Geus und Stubbe (de Geus und Stubbe 2007) erklären diese erhöhte Stressresistenz mit dem „Kompensationseffekt“ von Sport und Bewegung: Körperliche Aktivität und Stress wirken auf die gleichen gesundheitlichen Risikofaktoren ein, allerdings in entgegengesetzter Richtung. Die Autoren verdeutlichen dies am Beispiel der kardiovaskulären Risikofaktoren: Von chronischem Stress ist bekannt, dass er sich auf atherogene Faktoren wie LDLund HDL-Cholesterin, Triglyceride, Insulin und Blutdruck gesundheitsnegativ auswirkt (z. B. Burg und Pickering 2011), während von Sport und Bewegung auf genau die gleichen Risikofaktoren gesundheitspositive Effekte ausgehen (z. B. Hardman und Stensel 2003). Körperliche Aktivität wirkt hier den stressverursachten Gefährdungen der Herz-Kreislauf-Gesundheit kompensatorisch entgegen, und zwar unabhängig von anderen kognitiven, affektiven oder (stress-) physiologischen Wirkweisen der Sportaktivität.

Ganz ähnlich wird auch im Allostase-Modell von McEwen (McEwen 1998) die körperliche Aktivität als eine wirksame Strategie angesehen, um den negativen Auswirkungen der allostatischen Last auf die Gesundheit entgegenzuwirken. McEwen (McEwen 2002a) bemerkt hierzu: „If we counteract stress with a brisk walk or a visit to the health club, we can increase the odds in our favor. Exercise prevents the buildup of body fat, protects against cardiovascular disease, and reduces chronic pain and depression" (S. 66). Körperliche Aktivität stellt damit ein positives Gegengewicht zu negativen Stresseinflüssen auf die Gesundheit dar. 